\title{
A SPECTRAL PROPERTY FOR THE SYMBOLIC DYNAMICAL SYSTEM
}

\author{
K. Abodayeh \& A. Pokrovskii
}

Let $(X, \phi)$ be a topological dynamical system, that is, $X$ is a compact Hausdorff space and $\phi: X \rightarrow X$ is a homeomorphism. Recently, a great deal of attention has been paid to topological dynamical systems with the so called chaotic behaviour, see $[4,6]$ and the bibliography therein for a comprehensive discussion about what the chaotic behaviour means mathematically.

We can consider the linear operator $T_{\phi}$ defined by $T_{\phi}(f)=$ $f \circ \phi$, where $f$ is a function on $X$. If $(X, \phi)$ and $(Y, \psi)$ are topological dynamical systems, then they are isomorphic, and we write $\phi \cong \psi$, if there exists a homeomorphism $h: X \rightarrow Y$ such that $\phi=h^{-1} \psi h$. Clearly, $T_{\phi}=T_{h} T_{\psi} T_{h^{-1}}$ and therefore the spectral properties of $T_{\phi}$ are natural invariants with respect to the isomorphism relationship. In particular, if the spectral properties of $T_{\phi}$ are distinct from those of $T_{\psi}$, then we have $\phi \not \neq \psi$. It is interesting to investigate spectral properties of the operators $T_{\phi}$ that are typical for the dynamical systems $(X, \phi)$ with chaotic behaviour and this is the principal topic of the paper.

Consider the two-element group $\mathbf{Z}_{2}=\{0,1\}$ under addition modulo 2 and define the set $\Omega=\mathbf{Z}_{2}^{\mathbf{Z}}$; that is, $\Omega$ is the set of all doubly infinite sequences $\omega=\left(\omega_{n}\right)_{n \in \mathbf{Z}}$ with $\omega_{n} \in \mathbf{Z}_{2}$. Obviously $\Omega$ is compact when endowed with the product topology and operation. Define the shift transformation $\phi: \Omega \rightarrow \Omega$ by setting $\phi(\omega)=\left(\omega^{\prime}\right)$, where $\omega_{n}^{\prime}=\omega_{n+1}$ for all $n \in \mathbf{Z}$. The dynamical system $(\Omega, \phi)$ is called the symbolic dynamical system. This is a traditional first choice for the system exemplifying chaotic behaviour [3]. Moreover, sometimes a dynamical system $(X, \psi)$ is said to have chaotic behavior if there exists an invariant subset 
$Y \subseteq X$ such that the dynamical system $\left(Y,\left.\psi\right|_{Y}\right)$ is isomorphic to the dynamical system $(\Omega, \phi)[4]$.

\section{Theorem 1.}

(a) There exists a continuous function $g_{0} \in C(\Omega)$ such that for each complex $\lambda,|\lambda|=1$ the open ball $B\left(g_{0}, 1 / 8\right)$ does not intersect the range of $T_{\phi}-\lambda I$.

(b) 1 is the only eigenvalue of $T_{\phi}$.

The property (a) of the operator $T_{\phi}$ from Theorem 1 contrasts with the properties of the operator $T_{\phi}$ in $L_{2}(\mu)$ with the Bernoulli invariant measure $\mu$, [5]: for each $\lambda \in \mathbf{T}$ the range of the operator $\lambda I-T_{\phi}$ is dense in $L_{2}(\mu)$. Note also that the property mentioned in the theorem is invariant with respect to isomorphism:

Corollary 2. Let $(Y, \psi)$ be a topological dynamical system that is isomorphic to $(\Omega, \phi)$. Then the following spectral properties are valid:

(a) there exists a continuous function $\tilde{g}_{0} \in C(Y)$ such that for each $\lambda \in \mathbf{T}$, the open ball $B\left(\tilde{g}_{0}, 1 / 8\right)$ does not intersect the range of $T_{\psi}-\lambda I$

(b) 1 is the only eigenvalue of $T_{\psi}$.

The combination of spectral properties mentioned in Theorem 1 looks rather peculiar and may be typical only for dynamical systems which behave in a similar way to the hyperbolic homeomorphisms, [3]. This suggests that the following definition could be useful.

Definition. A topological dynamical systems $(X, \psi)$ is said to be $s$-chaotic if the operator $T_{\psi}: C(X) \rightarrow C(X)$ has no eigenvalues apart from 1 and, on the other hand, there exists an open ball $B\left(g_{0}, \varepsilon\right) \subset C(X)$ satisfying

$$
B\left(g_{0}, \varepsilon\right) \bigcap\left(\bigcup_{\lambda \in \mathbf{T}}\left(T_{\psi}-\lambda I\right)(C(X))\right)=\emptyset .
$$

Note that by Corollary 2 this definition is in line with the definition of chaotic behaviour as suggested in [4]. The tentative analysis shows that this definition does not apply to the dynamical systems 
$(Y, \psi)$ which are traditionally considered as 'non-chaotic' or have a non-chaotic component.

Example 3. As an example consider the irrational rotation $\psi_{\alpha}$ of a circle:

$$
e^{i \theta} \mapsto e^{i(\theta+2 \pi \alpha)}
$$

where $0 \leq \theta<2 \pi$ and $\alpha$ is a fixed irrational real number. This mapping is a typical example of an ergodic but not chaotic mapping. The spectrum of the operator $T_{\psi_{\alpha}}$ coincides with the unit circle of the complex plane, but its further properties contrast sharply with those mentioned in the definition of $s$-chaotic behaviour: the operator $T_{\psi_{\alpha}}$ has a countable number of eigenvalues $\lambda_{k}=e^{i 2 \pi \alpha k}, k=0, \pm 1, \pm 2, \ldots$ and for any $\lambda \neq \lambda_{k}, k=0, \pm 1$, $\pm 2, \ldots$ the range of the operator $T_{\psi_{\alpha}}-\lambda I$ is dense in $C(\mathbf{T})$.

On the other hand, the chaotic homeomorphisms different from the symbolic dynamical system are likely to be $s$-chaotic.

Problem. Prove that an algebraic toral automorphism [4] is $s$ chaotic.

This article is an extended abstract of a talk given in the 11th IMS meeting. More details, in particular the proof of Theorem 1, are given in [1].

The authors are grateful to Prof. P. E. Kloeden and Prof. G. J. Murphy for useful discussions.

\section{References}

[1] K. Abodayeh and A. Pokrovskii, Topological chaos: A spectral property for the shift on a sequence space, to appear, Nonlinear Functional Analysis, Theory, Methods, Applications,

[2] D. Lind and B. Marcus, An Introduction to Symbolic Dynamics and Coding. Cambridge University Press: Cambridge, 1995.

[3] R. Mane, Ergodic Theory and Differentiable Dynamics. SpringerVerlag: Berlin, 1987.

[4] D. Ruelle, Elements of Differentiable Dynamics and Bifurcation Theory. Academic Press: Boston, 1989. 
[5] Y. Sinai, Introduction to Ergodic Theory. Princeton University Press: New Jersey, 1976.

[6] P. Touhey, Chaos: the evolution of a definition, Irish Math. Soc. Bull. 40 (1998), 60-70.

K. Abodayeh

Department of Mathematics

University College Cork

Cork, Ireland

email: kamal@ucc.ie

A. Pokrovskii

Institute for Nonlinear Science

Department of Physics

University College Cork

Cork, Ireland

email: alexei@peterhead.ucc.ie 